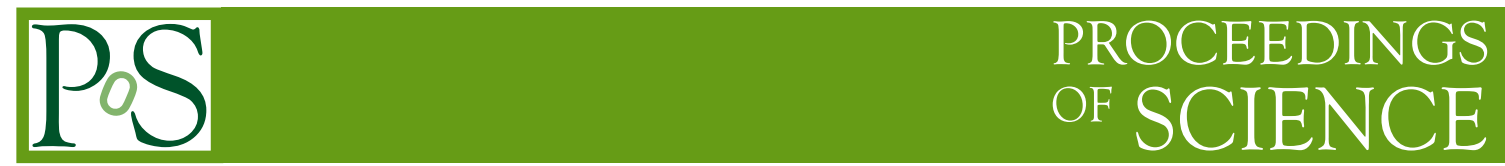

\title{
Flow and correlations results from CMS
}

\author{
Damir Devetak* \\ CMS Collaboration \\ E-mail: damir.devetak@cern.ch
}

The observation of long-range two-particle correlations in high energy heavy ion collisions opens the opportunities of exploring novel QCD dynamics in quark gluon plasma (QGP), the hot dense matter created in heavy ion collisions. In particular, such correlations are sensitive to the degree of thermalization in the system. We present selected results from non-identified and identified two-particle and multi-particle correlations in $\mathrm{PbPb}$ collisions, as well as in lighter systems.

The European Physical Society Conference on High Energy Physics 22-29 July 2015

Vienna, Austria

*Speaker. 


\section{Introduction}

Ultra-relativistic heavy ion collisions are a ideal terrain for exploring strongly interacting matter under extreme conditions. A stepping stone in high energy physics is the discovery of QGP at RHIC (Relativistic Heavy-Ion Collider) [1], a new medium defined as thermally equilibrated state of matter in which quarks and gluons are deconfined from hadrons [2]. Two observables that advocate directly the existence of Quark-Gluon Plasma are measurements of flow and the suppression of high $p_{T}$ hadrons. The flow, as a manifestation of non-uniform emission of final state particles, can be described successfully by relativistic hydrodynamics treating the QGP as almost a perfect liquid. Two-particle and multi-particle correlations have been used extensively at the CMS experiment in quantifying the spacial anisotropy through measurements of the flow signal $v_{n}$ with identified and non-identified reconstructed particles.

\section{Multi-particle correlations in $\mathrm{PbPb}$ and $\mathrm{pPb}$}

Dihadron correlations are a robust tool in handling the effects of particle production in relativistic heavy ion collisions. One significant effect that was observed in two-particle correlations is the presence of a near side $(\Delta \phi \sim 0)$ "ridge-like" structure. This long range correlation was first measured at the STAR experiment $[3,4]$ and than extensively investigated at LHC energies $[5,6,7,8]$. In the case of symmetrical nucleus-nucleus (AA) collisions this ridge-like structure can be explained by higher order harmonics predominantly by the triangular $v_{3}$ flow [9]. However, these long-range correlations have also been observed in smaller systems, like proton-proton (pp) [10] and proton-lead (pPb) $[11,12]$ collisions for high multiplicity case at the LHC. The question then arises if this long-range correlations, for smaller systems, can also be put in the context of hydrodynamic models. One way of inferring this is to investigate multi-particle correlations since, from a hydrodynamical point of view, all multi-particle azimuthal correlations stem from anisotropic collective expansion of the created medium. In figure 1 the second-order Fourier harmonic $v_{2}$, averaged over $0.3<p_{T}<3.0 \mathrm{GeV} / \mathrm{c}$, is plotted as a function of multiplicity $N_{\mathrm{trk}}^{\text {offine }}$ for $\mathrm{PbPb}$ (left) and $\mathrm{pPb}$ (right). With open symbols results of $v_{2}$ from two and four-particle correlations from CMS [13] are presented. As an extension of this analysis higher order correlations were calculated, the six- $\left(v_{2}\{6\}\right)$ and the eight- $\left(v_{2}\{8\}\right)$ particle case, and also the Lee-Yang zero which represents the all-particle correlation method. For both the symmetrical $\mathrm{PbPb}$ and asymmetrical $\mathrm{pPb}$ system, the $v_{2}$ falls within the $\pm 10 \%$ difference range for all the methods $\left(v_{2}\{4\}, v_{2}\{6\}, v_{2}\{6\}\right.$ and LYZ) a result that favours the collective nature of the analysed systems. Comparing the two-particle $v_{2}\{2\}$ results with higher order correlations we see consistent higher values which can be explained by hydrodynamical models as a consequence of event-by-event participant geometry fluctuations.

\section{Two-particle correlations of strange hadrons}

CMS conducted measurements of two-particle angular correlations between identified strange hadrons $\left(K_{S}^{0}\right.$ and $\left.\Lambda / \bar{\Lambda}\right)$ and unidentified charged particles in $\mathrm{PbPb}$ and $\mathrm{pPb}$ collisions. The reconstruction technique for $K_{S}^{0}$ and $\Lambda / \bar{\Lambda}$ candidates (generally referred to as $V^{0} s$ ) is described in [14]. Using the two-particle method the construction of the corresponding correlation function follows 


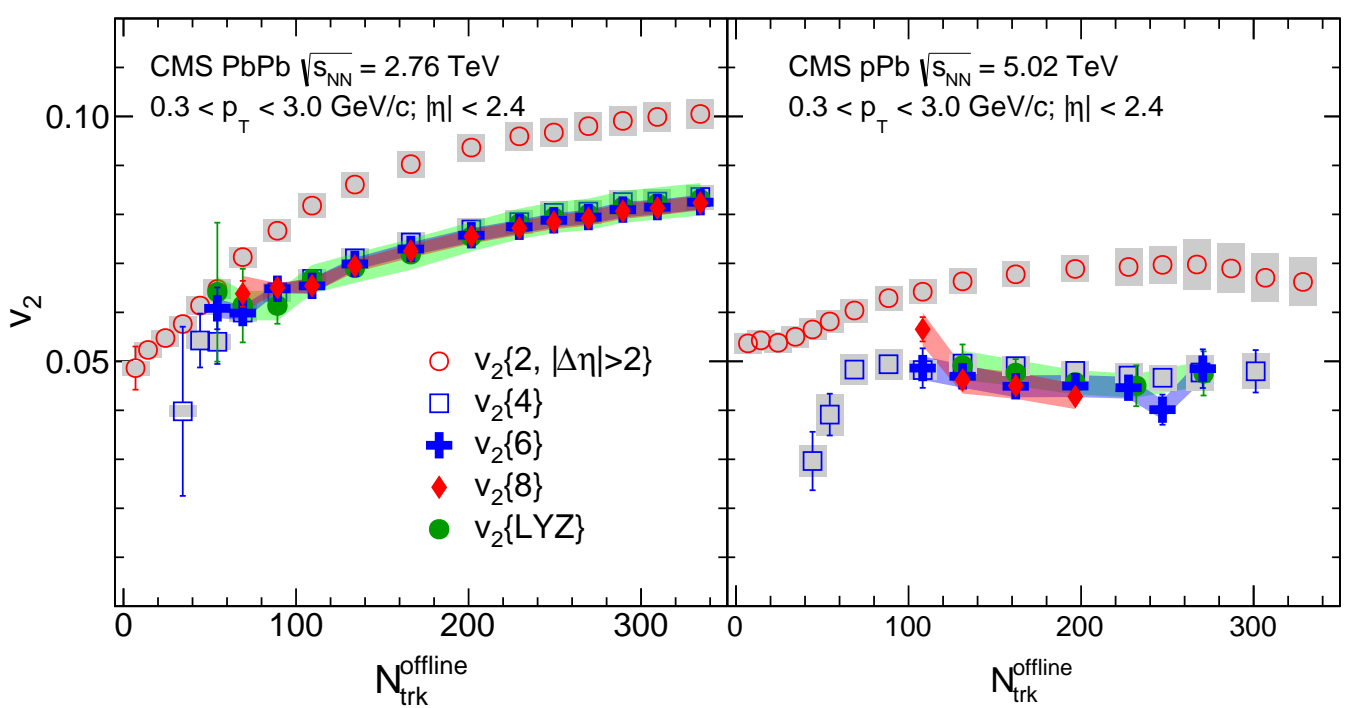

Figure 1: Values of $v_{2}$ obtained from 6-particle (blue cross), 8-particle (red diamond) cumulants, and LYZ (green filled circle) method, averaged over particle $p_{T}$ range of $0.3-3.0 \mathrm{GeV} / \mathrm{c}$, as a function of multiplicity $N_{\text {trk }}^{\text {offline }}$ in $\mathrm{PbPb}$ at $\sqrt{s_{N N}}=2.76 \mathrm{TeV}$ (left) and $\mathrm{pPb}$ at $\sqrt{s_{N N}}=5.02 \mathrm{TeV}$ (right). Open data points are published two-particle and four-particle $v_{2}$ results from [13]. Shaded areas denote systematic uncertainties.

the same methodology as in $[15,16]$. The azimuthal two-particle harmonics $V_{n \Delta}$ of $K_{S}^{0}$ and $\Lambda / \bar{\Lambda}$ are extracted through the Fourier decomposition of the correlation function averaged over $|\Delta \eta|>2$ (in order to remove short-range correlations such as jets) as was done in [14]. Using the two-particle Fourier coefficients the single-particle harmonics are calculated as,

$$
v_{n}\left(p_{T}^{V_{0}}\right)=\frac{V_{n \Delta}\left(p_{T}^{V_{0}}, p_{T}^{r e f}\right)}{\sqrt{V_{n \Delta}\left(p_{T}^{r e f}, p_{T}^{r e f}\right)}}, \quad n=2,3 .
$$

Here the $p_{T}^{r e f}$ is the reference bin for the range $0.3<p_{T}^{r e f}<3.0 \mathrm{GeV} / \mathrm{c}$ to minimize correlations from jets at higher $p_{T}$. Figure 2 shows measured $v_{2}$ values of $K_{S}^{0}$ and $\Lambda / \bar{\Lambda}$ and inclusive charged

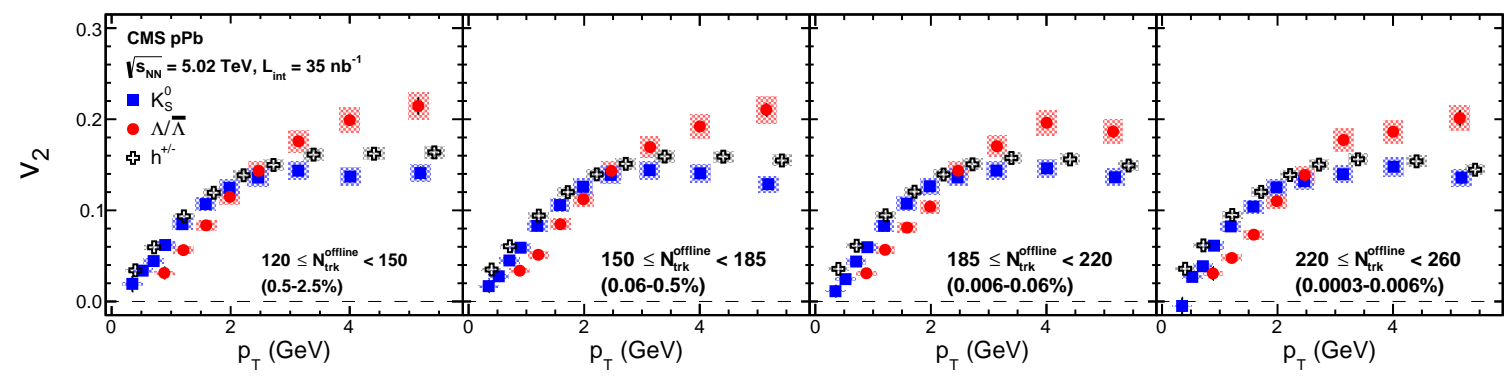

Figure 2: $v_{2}$ results for $K_{S}^{0}$ (filled squares), $\Lambda / \bar{\Lambda}$ (filled circles) and inclusive charged particles (open crosses) as a function of $p_{T}$ for four multiplicity ranges in $\mathrm{pPb}$ collisions at $\sqrt{s_{N N}}=5.02 \mathrm{TeV}$.

particles for $\mathrm{pPb}$ collisions. In the $p_{T} \preceq 2 \mathrm{GeV} / \mathrm{c}$ domain for all multiplicity ranges, the $v_{2}$ values for $K_{S}^{0}$ particles are larger than those of $\Lambda / \bar{\Lambda}$ with inclusive charged particles $v_{2}$ values being above the $K_{S}^{0}, \Lambda / \bar{\Lambda}$ cases. For higher $p_{T}$ values the mass ordering reverses for $K_{S}^{0}$ and $\Lambda / \bar{\Lambda}$ now with inclusive 
charged particles $v_{2}$ values falling between the values of the two identified strange hadron species. Comparing the $\mathrm{PbPb}$ and $\mathrm{pPb}$ case, even though one observes qualitatively a similar particle-species mass ordering of $v_{2}$, the mass ordering effect is found to be less clear in $\mathrm{PbPb}$ data than in $\mathrm{pPb}$ data for all multiplicity ranges [14]. In addition, the scaling effect was investigated, diving the $v_{2}$ by the number of constituent quarks and plotting the values as a function of transverse kinetic energy per quark $\left(K E_{T}\right)$ for $\mathrm{pPb}$ and $\mathrm{PbPb}$. Figure 3 shows the $v_{2} / n_{q}$ values for $K_{S}^{0}, \Lambda / \bar{\Lambda}$ particles in $\mathrm{pPb}$ collisions. After introducing the scaling by the number of quarks the $v_{2}$ values for both particle species approximately fall on the same curve. This effect is conjectured to be connected with the quark recombination $[17,18]$, which postulates that collective flow is developed among constituent quarks first before they combine into final-state hadrons.

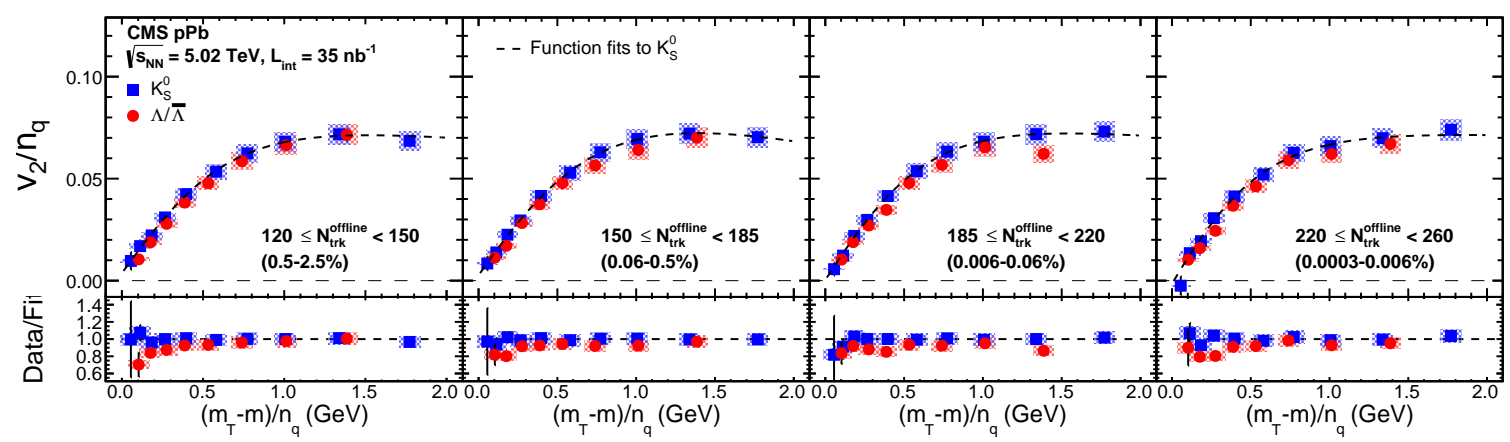

Figure 3: Ratio of $v_{2} / n_{q}$ for $K_{S}^{0}$ (filled squares), $\Lambda / \bar{\Lambda}$ (filled circles) particles as a function of $K E_{T} / n_{q}$ in $\mathrm{pPb}$ collisions at $\sqrt{s_{N N}}=5.02 \mathrm{TeV}$.

\section{Factorization breaking effect}

Dealing with two-particle correlations a common assumption, in a pure hydrodynamical flow picture, is the effect of factorization. This means that the two-particle harmonic $V_{n \Delta}\left(p_{T}^{a}, p_{T}^{b}\right)$ is equal to the product of single particles anisotropies $v_{n}\left(p_{T}^{a}\right) \times v_{n}\left(p_{T}^{b}\right)(n>1)$ i.e. it factorizes, explaining any deviation from this equality with contributions from non-flow effects. However, recent studies from hydrodynamics $[19,20,21]$ show that this effect can follow completely from a pure flow picture induced via $p_{T}$-dependent event plane angle a dependence caused by initial state fluctuations within nuclei. This is illustrated from the following equation showing the relation between the harmonics,

$$
V_{n \Delta}\left(p_{T}^{a}, p_{T}^{b} ; \eta^{a}, \eta^{b}\right)=\left\langle v_{n}\left(p_{T}^{a}\right) v_{n}\left(p_{T}^{b}\right) \cos \left[n\left(\Psi_{n}\left(p_{T}^{a}, \eta^{a}\right)-\Psi_{n}\left(p_{T}^{b}, \eta^{b}\right)\right)\right]\right\rangle .
$$

From the last equation we can conclude that only when the event-plane angle is a global phase (no $p_{T}$ and $\eta$ dependence) we observe factorization. CMS conducted a detailed analysis [22] on the effect of factorization breaking focusing on both the $p_{T}$ and $\eta$ dependence of the event-plane angle. In order to quantify the effect for $p_{T}$ case one uses the ratio $r_{n}$ as in [22],

$$
r_{n}=\frac{V_{n \Delta}\left(p_{T}^{a}, p_{T}^{b}\right)}{\sqrt{V_{n \Delta}\left(p_{T}^{a}, p_{T}^{a}\right) V_{n \Delta}\left(p_{T}^{b}, p_{T}^{b}\right)}} .
$$




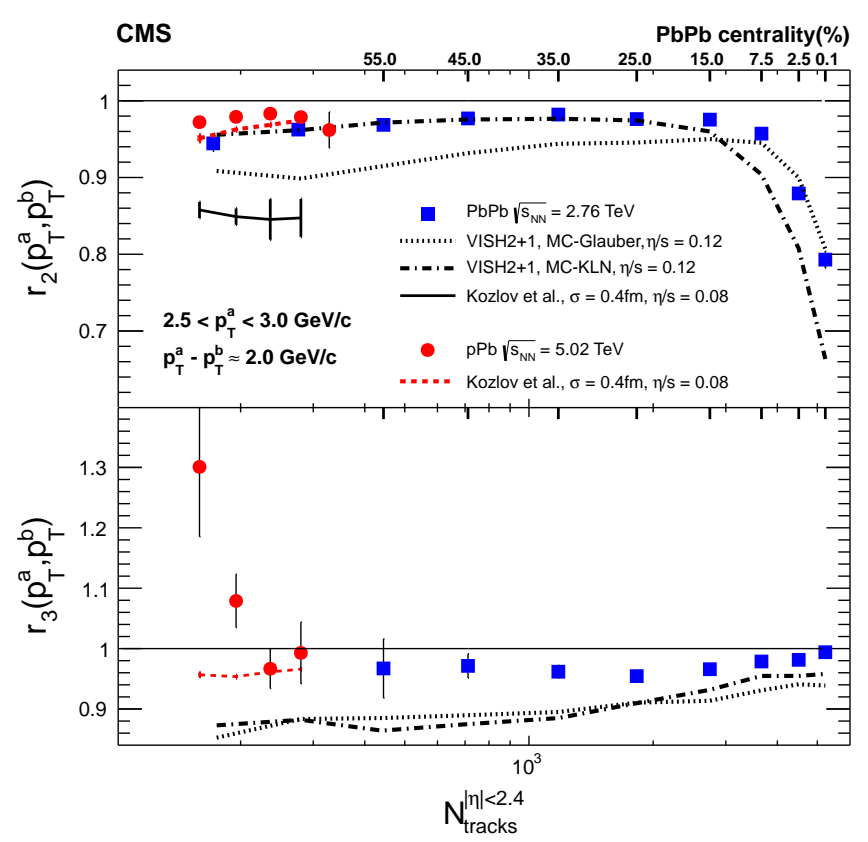

Figure 4: Ratio $r_{n}$ for the elliptic and triangular case in $\mathrm{PbPb}$ collisions at $\sqrt{s_{N N}}=2.76 \mathrm{TeV}$ and in $\mathrm{pPb}$ collisions at $\sqrt{s_{N N}}=5.02 \mathrm{TeV}$ as a function of centrality (multiplicity). Dashed lines show predictions of hydrodynamical models [20, 21].

In a hydrodynamical picture the ratio can only be $r_{n} \leq 1$, factorization breaking referring to values of $r_{n}$ below unity, with $r_{n}>1$ implying non-collective contributions. Figure 4 shows values of $r_{n}$ for the elliptic and triangular case for $\mathrm{PbPb}$ and $\mathrm{pPb}$ collisions. The ratio is plotted versus centrality (multiplicity) including comparisons with hydro models predictions [20, 21]. In the case of $\mathrm{PbPb}$ collisions, looking from left to right, increasing centrality we see a steep drop of $r_{2}$ value going to around 0.8 (20\% below unity) for the most central collisions $(0-0.2 \%)$ where the initial state fluctuations are most dominant. Lowering the centrality and looking at peripheral collisions we observe that the effect is small and is on the level of 3-5\%. Comparing the $r_{2}$ values for high multiplicity $\mathrm{pPb}$ collisions we see that it is similar in intensity with peripheral $\mathrm{PbPb}$ collisions. The triangular case $r_{3}$ shows that factorization holds better for this higher flow order. Using the hydrodynamical predictions for different initial-state conditions, we conclude that quantitatively they describe the experimental data well.

In addition, the longitudinal $(\eta)$ event-plane angle dependence was investigated in $\mathrm{PbPb}$ and $\mathrm{pPb}$ collisions. The ratio $r_{n}$ for the $\eta$ case is defined as,

$$
r_{n}\left(\eta^{a}, \eta^{b}\right)=\frac{V_{n \Delta}\left(-\eta^{a}, \eta^{b}\right)}{V_{n \Delta}\left(\eta^{a}, \eta^{b}\right)} \approx e^{-2 F_{n}^{\eta} \eta^{a}} .
$$

As for the $p_{T}$ case, if the event-plane angle has longitudinal fluctuations the ratio $r_{n}\left(\eta^{a}, \eta^{b}\right)$ should have values below unity. The harmonics $V_{n \Delta}\left(\eta^{a}, \eta^{b}\right)$ are calculated in the same way as in the case of $V_{n \Delta}\left(p_{T}^{a}, p_{T}^{b}\right)$, now correlating tracks from two different $\left(\eta^{a}, \eta^{b}\right)$ bins. Here, particle $a$ is selected from the range $|\eta|<2.4$ and $0.3<p_{T}<3.0 \mathrm{GeV} / \mathrm{c}$, while particle $b$ is selected from the Hadron Forward Towers ( total coverage of $2.9<\eta<5.2$ ) with the energy exceeding $1 \mathrm{GeV}$. In this way, 


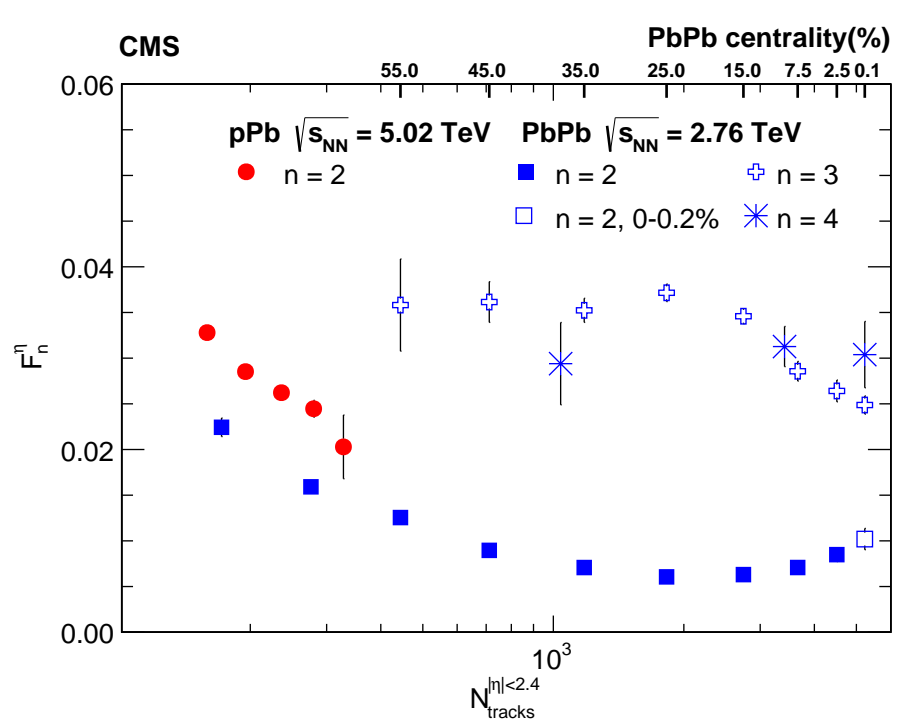

Figure 5: The $F_{n}^{\eta}$ parameter as a function of multiplicity in $\mathrm{PbPb}$ collisions at $\sqrt{s_{N N}}=2.76 \mathrm{TeV}$ for $\mathrm{n}=2-4$ and $\mathrm{pPb}$ collisions at $\sqrt{s_{N N}}=5.02 \mathrm{TeV}$ for $\mathrm{n}=2$.

paring up tracks $(a, b)$ from two sub-detectors we can apply a large $\Delta \eta$ cut between them and suppress the non-flow contributions. Observing differential values of $r_{n}\left(\eta^{a}, \eta^{b}\right)$ [22] we conclude that the ratio is independent of $\eta^{b}$, for $\eta^{a}$ ranges where the long-range cut $(|\Delta \eta|>2)$ is valid. This can be used to introduce a parametrization of the ratio 4.3, with the assumption that relative fluctuations between two event plane angles depend only on their pseudorapidity difference. Figure 5 shows values of parameter $F_{n}^{\eta}$ as a function of multiplicity, in $\mathrm{pPb}$ collisions for $\mathrm{n}=2$ and $\mathrm{PbPb}$ collisions for $\mathrm{n}=2-4$. The minimum for $F_{2}^{\eta}$ is around mid-central $(20 \%) \mathrm{PbPb}$ collisions, and increases significantly for more peripheral $\mathrm{PbPb}$ events and also for $\mathrm{pPb}$ events. For the most central $\mathrm{PbPb}$ collisions, the $F_{2}^{\eta}$ value increases slightly, although the $r_{2}$ data for $0-0.2 \%$ centrality are not well described by 4.3 . For similar multiplicities, magnitude of $F_{2}^{\eta}$ in $\mathrm{pPb}$ is significantly larger than those in $\mathrm{PbPb}$. In $\mathrm{PbPb}$ collisions, a much stronger longitudinal factorization breakdown is seen for higher-order harmonics in comparison with $\mathrm{n}=2$, as shown by the $F_{3}^{\eta}$ and $F_{4}^{\eta}$ parameters.

\section{Summary}

CMS conducted detailed studies in $\mathrm{PbPb}$ collisions at $\sqrt{s_{N N}}=2.76 \mathrm{TeV}$ and $\mathrm{pPb}$ collisions at $\sqrt{s_{N N}}=5.02 \mathrm{TeV}$ using two- and multi-particle correlations with non-identified and identified particles. The observed long-range correlation ("ridge") in $\mathrm{pPb}$ is investigated with higher order cumulants, the six- $\left(v_{2}\{6\}\right)$ and the eight- $\left(v_{2}\{8\}\right)$ particle case and also the Lee-Yang zero which represents the all-particle case. The Fourier coefficient $v_{2}$ shows no dependence on the number of correlated particles with significant non-zero values favouring a hydrodynamical picture even for lighter systems. Two particle correlations were used in extracting single-particle anisotropy for reconstructed strange hadrons $K_{S}^{0}, \Lambda / \bar{\Lambda}$ demonstrating important effects like "scaling" and mass ordering of $v_{2}$. Finally, CMS investigated the effect of factorization breaking caused by the $p_{T}$ and $\eta$ dependent event-plane angle. The results show a clear presence of this effect for both the 
transversal momentum and longitudinal case which is caused by initial state fluctuations within the nuclei.

\section{References}

[1] Proceedings of the 15th International Conference on Ultrarelativistic Nucleus-Nucleus Collisions (QM 2001), Stony Brook, New York, January, 15-20, 2001, Nucl. Phys. A 698 (2002).

[2] J. Adams et al. [STAR Collaboration], Nucl. Phys. A 757 (2005) 102.

[3] J. Adams et al. [STAR Collaboration], Phys. Rev. Lett. 95, 152301 (2005).

[4] B. I. Abelev et al. [STAR Collaboration], Phys. Rev. C80, 064912 (2009).

[5] S. Chatrchyan et al.[CMS Collaboration], JHEP 1107 (2011) 076.

[6] S. Chatrchyan et al. [CMS Collaboration], Eur. Phys.J.C 72 (2012) 2012.

[7] K. Aamodt et al. [ALICE Collaboration], Phys. Lett. B 708 (2012) 249.

[8] G. Aad et al. [ATLAS Collaboration], Phys. Rev. C 86 (2012) 014907.

[9] B. Alver and G. Roland, Phys. Rev. C 81, 054905 (2010).

[10] V. Khachatryan et al. [CMS Collaboration], JHEP 1009 (2010) 091.

[11] S. Chatrchyan et al. [CMS Collaboration], Phys. Lett. B 718 (2013) 795.

[12] B. Abelev et al. [ALICE Collaboration], Phys. Lett. B 719 (2013) 29.

[13] S. Chatrchyan et al. [CMS Collaboration], Phys. Lett. B 724 (2013) 213.

[14] S. Chatrchyan et al. [CMS Collaboration], PLB 742 (2015) 200.

[15] S. Chatrchyan et al. [CMS Collaboration], JHEP07 (2011) 076.

[16] S. Chatrchyan et al. [CMS Collaboration], Eur. Phys. J. C 72 (2012).

[17] D. Molnar and S. A. Voloshin, Phys. Rev. Lett. 91 (2003) 092301.

[18] V. Greco, C. Ko, and P. Levai, Phys. Rev. Lett. 90 (2003) 202302.

[19] F. G. Gardim, F. Grassi, M. Luzum, and J.-Y. Ollitrault, Phys. Rev. C 87, (2013) 031901.

[20] U. Heinz, Z. Qiu, and C. Shen, Phys. Rev. C 87, (2013) 034913.

[21] Igor Kozlov et al., arXiv:1405.3976.

[22] S. Chatrchyan et al. [CMS Collaboration], Phys. Rev. C 92, 034911. 